\section{Cahiers de Narratologie}

Analyse et théorie narratives

39 | 2021

L'héritage de Ricoeur : du récit à l'expérience

\title{
Paul Ricœur et la narratologie : un dialogue croisé
}

Ioana Vultur

\section{OpenEdition}

Journals

Édition électronique

URL : https://journals.openedition.org/narratologie/12073

DOI : 10.4000/narratologie. 12073

ISSN : 1765-307X

Éditeur

LIRCES

Référence électronique

loana Vultur, « Paul Ricœur et la narratologie : un dialogue croisé », Cahiers de Narratologie [En ligne], 39 | 2021, mis en ligne le 03 juin 2021, consulté le 21 juillet 2021. URL : http://

journals.openedition.org/narratologie/12073; DOI : https://doi.org/10.4000/narratologie.12073

Ce document a été généré automatiquement le 21 juillet 2021.

Article L.111-1 du Code de la propriété intellectuelle. 


\title{
Paul Ricœur et la narratologie : un dialogue croisé
}

\author{
Ioana Vultur
}

1 Roland Barthes soulignait dans son Introduction à l'analyse structurale la grande variété des récits que l'on rencontre

dans le mythe, la légende, la fable, le conte, la nouvelle, l'épopée, l'histoire, la tragédie, le drame, la comédie, la pantomime, le tableau peint (que l'on pense à Sainte Ursule de Carpaccio), le vitrail, le cinéma, les comics, le fait divers, la conversation. De plus, sous ces formes presque infinies, le récit est présent dans

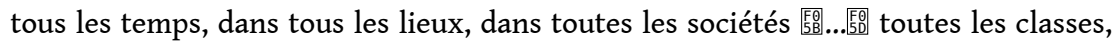
tous les groupes humains ont leurs récits, et bien souvent ces récits sont goûtés en commun par des hommes de culture différente, voire opposée (Barthes $1966: 828$ ).

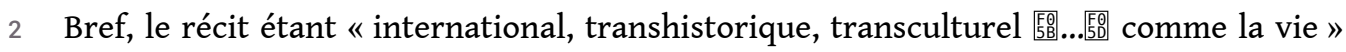
(1966: 828), le but de la narratologie devait être, selon Barthes, de réduire cette diversité à un invariant universel, une sorte de modèle, une structure profonde commune à tous les récits.

Dans un contexte dans lequel les sciences humaines et sociales (études littéraires, histoire, anthropologie) étaient encore dominées par le structuralisme, Ricoeur est l'un des seuls qui, en France, entre en débat avec le structuralisme au nom d'une conception herméneutique des études littéraires et des sciences sociales. Dans son ouvrage Temps et récit, il met en évidence l'importance du récit et développe une théorie visant à dépasser les limites de la méthode structurale. Il analyse le mode de construction du temps historique dans l'historiographie, il met en évidence le rôle fondamental du récit de fiction dans la configuration du temps et il situe la problématique du temps dans une perspective plus vaste, philosophique, étudiant les apories d'une philosophie du temps vécu. Si le structuralisme avait déchronologisé le récit pour faire apparaître « la logique narrative sous-jacente au temps narratif», Ricoeur en revanche souligne le caractère temporel du récit qui est indissociable de notre façon de vivre dans le monde, ce qui l'amène à affirmer que « le temps devient temps humain dans la mesure où il est articulé de manière narrative » et qu' " en retour le récit est significatif dans la mesure où il dessine les traits de l'expérience temporelle » (Ricoeur $1983: 17$ ). 
Dans un premier temps, je montrerai que grâce à Ricoeur, il y a eu une renaissance des études sur le récit, mais que ce tournant narratif avait déjà été amorcé dans les sciences humaines. Je partirai de la théorie de la triple mimèsis de Ricoeur dans laquelle il définit la narrativité à partir de la mise en intrigue, tout en montrant que cette dernière est ancrée dans l'expérience et qu'elle refigure l'expérience. Je m'intéresserai ensuite à la relation entre mimèsis II et mimèsis III, telle qu'elle est décrite dans Temps et récit II et je montrerai comment Ricoeur entre en débat avec la narratologie structuraliste, non seulement pour critiquer sa position, mais aussi pour l'intégrer à son propre modèle. Je montrerai enfin que le modèle de Ricoeur a eu un impact sur la narratologie postclassique. Ainsi, la narratologie contemporaine, surtout la narratologie cognitive, est partie d'une critique du modèle de la narratologie structuraliste et a développé en quelque sorte des possibilités qui se trouvaient in nuce dans Temps et récit. Le monde du texte est désormais considéré comme ouvert vers le lecteur, car la narratologie cognitive s'intéresse aux relations entre récit et esprit. La notion centrale d'« expérientialité" introduite par Monika Fludernik reprend la discussion de l'articulation entre intrigue et expérience dans le cadre de la narratologie.

\section{La question de la narrativité}

5 La publication de Temps et récit de Paul Ricoeur a engendré un renouveau de l'intérêt pour la question de la narrativité à un moment où le structuralisme était entré en crise (voir Rimmon-Kenan 1989: 157). Il faut cependant préciser que l'intérêt de Ricoeur pour le récit s'inscrit dans un contexte plus global, qui voit les sciences humaines et sociales dans leur ensemble accorder une importance nouvelle à la narrativité, à tel point qu'on peut parler d'un tournant narratif. Jerome Bruner dans le domaine de la psychologie, Clifford Geertz en anthropologie, Hayden White pour l'historiographie, ont tous souligné l'importance de la narrativité pour les sciences humaines et sociales. Dans le cas de la microhistoire, cette revalorisation du récit s'accompagne d'un déplacement de l'intérêt sur l'expérience individuelle, sur les cas singuliers, aux dépens de l'histoire des collectivités (Giovanni Levi, Carlo Ginzburg). La mise en avant de l'expérience et des histoires de vie s'observe aussi en sociologie. Goffman s'intéresse ainsi aux « cadres de l'expérience », Berger et Luckmann développent une sociologie de la compréhension partant de la théorie de Schütz, qui met en avant la position de l'acteur social qui vit l'expérience (Schütz 1932).

6 Il faut noter par ailleurs que l'intérêt de Ricoeur pour le récit part d'un souci philosophique : selon lui, les philosophes n'ont pas réussi à dépasser l'aporie entre le temps intérieur, c'est-à-dire le temps subjectif de la conscience, et le temps extérieur, le temps cosmique, et à penser ces deux versants du temps ensemble. Si donc Ricœur accorde un rôle cognitif très important au récit, c'est parce que, selon lui, seul le récit peut faire la médiation entre ces deux temps : le temps ne se laisse pas conceptualiser mais seulement raconter.

7 La conception du récit de Ricœur repose sur une réinterprétation du concept de mimèsis d'Aristote. Chez Aristote, l'intrigue (muthos) est définie comme représentation ou mimèsis de l'action. Par « intrigue ", Aristote entendait plus précisément l'« agencement des faits » ou, pour reprendre les termes de Ricœur, l'« opération de configuration » opérée par la narration. Pour mettre en évidence qu'il s'agit d'une opération 
dynamique, Ricœur préfère utiliser l'expression de mise en intrigue, qui insiste davantage sur le caractère processuel de la configuration.

Ricœur met en évidence la fonction médiatrice de la mise en intrigue à trois niveaux. Premièrement, elle transforme des événements décousus en une totalité intelligible, en une figure : elle " tire une histoire sensée de - un divers d'événements et d'incidents » ou «transforme les événements ou incidents en - une histoire » (Ricoeur 1983 : 127). Le récit est donc une manière de rendre intelligible l'action humaine, en en dégageant la structure dynamique et l'unité. Deuxièmement, la mise en intrigue " compose ensemble des facteurs aussi hétérogènes que des agents, des buts, des moyens, des interactions, des circonstances, des résultats inattendus, etc. " (Ricoeur 1983:127) et c'est pourquoi Ricœur la définit comme "concordance-discordance ». Troisièmement, elle configure le temps : elle opère une "synthèse de l'hétérogène " (Ricoeur 1983 :128). Le récit a donc non seulement une dimension épisodique ou chronologique mais aussi un aspect configurant.

9 À la différence d'Aristote, qui avait réservé le terme de mimèsis à la fiction, Ricœur l'utilise pour désigner la structure qui est commune au récit historique et au récit de fiction. Il étend donc le modèle de mise en intrigue à toute composition narrative (Ricoeur 1983 : 73). Il montre que même l'historiographie antinarrative n'arrive pas à se débarrasser totalement du récit et de la mémoire. Cette impossibilité de l'histoire de se couper du récit est due selon Ricœur au fait qu'elle rend compte d'événements qui ont eu lieu dans le temps et que la forme sous laquelle les hommes comprennent de tels événements est le récit. Si l'histoire a une dimension narrative, c'est parce qu'elle est une façon de connaître et de comprendre le monde qui est ancrée dans l'expérience. Le récit apparaît ainsi comme une interface entre l'histoire comme science et l'expérience de l'histoire (Vultur 2017).

10 La notion de mimèsis de Ricœur est aussi plus large que celle d'Aristote, parce que Ricœur distingue trois niveaux de la mimèsis : la mimèsis I, c'est-à-dire la narrativité inchoative de l'action ; la mimèsis II, c'est-à-dire la mise en intrigue proprement dite ; et la mimèsis III, à savoir la refiguration par la réception de l'œuvre. L'intrigue au sens restreint du terme fait donc la médiation entre la mimèsis I, c'est-à-dire la précompréhension du monde de l'action, de sa sémantique et de sa symbolique, et la mimèsis III, c'est-à-dire la refiguration de l'œuvre grâce à l'acte de lecture. Dans ce modèle, l'expérience intervient à la fois en amont et en aval du texte. La mimèsis I ellemême est déjà structurée narrativement car, selon Ricœur, il y a une narrativité inhérente au monde de l'action. Ricœur parle d'« histoires non encore racontées » qui «demandent à être racontées» (Ricoeur 1983 : 141). L'expérience intervient aussi en aval, car les œuvres nous permettent de faire des expériences nouvelles, inédites, qui peuvent nous amener à une meilleure compréhension de nous-mêmes, des autres et du monde.

11 Mais pour Ricœur l'importance de la narrativité ne se limite pas à mettre à notre disposition des modèles d'expérience. Elle constitue le principe structurant de notre identité : en tant que «structure d'expérience commune au récit de fiction et au récit factuel »(Ricoeur 1985 : 442), elle est produite par la refiguration du temps par le récit, qu'il soit factuel ou fictif. C'est elle qui est au cœur de "la troisième relation mimétique » qui « se définit par l'identité narrative d'un individu ou d'un peuple, issu de la rectification sans fin d'un récit antérieur par un récit ultérieur, et de la chaîne des 
refigurations qui en résulte» (Ricoeur 1985 : 446). Il souligne que « la connaissance de soi est une interprétation » et que

l'interprétation de soi, à son tour, trouve dans le récit, parmi d'autres signes et symboles, une médiation privilégiée, - cette dernière emprunte à l'histoire autant qu'à la fiction, faisant de l'histoire d'une vie une histoire fictive ou, si l'on préfère, une fiction historique, comparable à ces biographies de grands hommes où se mêlent l'histoire et la fiction. (Ricoeur $1988: 295$ )

Si Ricœur se réfère à la fois à l'identité narrative des individus et à l'identité collective des communautés, c'est parce que selon lui on ne peut pas se comprendre soi-même de façon directe, mais uniquement par les œuvres de la culture (récits, symboles).

Ricœur est revenu à la notion d'identité narrative dans Soi-même comme un autre, où il montre qu'elle fait la médiation entre l'identité-mêmeté et l'identité-ipséité, articulant identité et altérité. Il s'intéresse ici en particulier à la façon dont la fiction donne lieu à des variations imaginatives sur l'identité. Si la notion d'identité narrative a été contestée par Galen Strawson, l'importance de la structuration narrative de l'identité humaine semble être confirmée par les recherches en psychologie cognitive consacrées à la mémoire et aux liens entre dysnarrativités et troubles de l'identité (voir Vultur 2017 : 112-115 ; Schaeffer 2020 : 77-98). Dans un travail pionnier, Young et Saver ont ainsi mis en évidence l'existence de quatre formes de dysnarrativité (Young et Saver : 72-84). Une première forme de dysnarrativité est la «narration arrêtée ». Dans cette pathologie, la mémoire des patients s'arrête à la date où est survenue leur lésion cérébrale. L'image que ces patients ont d'eux-mêmes reste immuable. Dans le cas de la "narration incontrôlée ", les patients racontent plusieurs histoires contradictoires en réponse à une même question. Une troisième forme de dysnarrativité est la «sousnarration ». Les individus qui en sont atteints conservent une mémoire autobiographique intacte mais sont incapabales de construire des scénarios contrefactuels. Une quatrième forme de dysnarrativité est la «dé-narration». Les patients qui souffrent de ce dysfonctionnement sont incapables de donner un compterendu narratif de leurs expériences, désirs et actions, ce qui les rend incapables de s'engager dans des actions intentionnelles planifiées par eux-mêmes. On voit que dans tous ces cas, la dysnarrativité se traduit par un important trouble de l'identité personnelle.

\section{De la narratologie à l'herméneutique : de l'intrigue à l'expérience}

14 C'est dans Temps et récit II, qui est consacré au récit fictionnel, que Ricœur entre en débat avec la narratologie: il commente en particulier la morphologie des contes de Propp, la Logique du récit de Claude Bremond, la sémiotique narrative de Greimas et Figures III de Genette.

Dans le chapitre intitulé «Les jeux avec le temps ", il reprend la distinction entre temps du raconter (Erzählzeit) et temps raconté (erzählte Zeit), introduite par Günther Müller et développée par Gérard Genette. Aux catégories immanentes au texte proposées par Genette, à savoir l'énonciation à laquelle correspond le temps du raconter (Erzählzeit) et l'énoncé auquel correspond le temps raconté (erzählte Zeit), Ricœur ajoute la catégorie de "monde du texte" à laquelle correspond selon lui « une expérience fictive $d u$ temps » (p. 143). Le jeu avec le temps, c'est-à-dire le rapport entre temps du raconter et 
temps raconté a donc un enjeu qui consiste en une expérience fictive du temps qui a pour horizon un monde imaginaire (Ricoeur $1984: 189$ ).

La conception de Ricœur s'oppose à la fois à la conception intentionnaliste classique, qui cherche l'intention de l'auteur derrière le texte, et à la conception structuraliste, qui cherche le sens dans l'immanence du texte. C'est la notion de "monde du texte " qui devient décisive pour lui. Il souligne ainsi que « ce qui est [...] à interpréter dans un texte, c'est une proposition de monde, d'un monde tel que je puisse l'habiter pour y projeter un de mes possibles les plus propres. C'est ce que j'appelle le monde du texte, le monde propre à ce texte unique » (Ricoeur 1986 : 128).

Dans la conception de Ricœur, « une œuvre peut être à la fois être close sur elle-même quant à sa structure et ouverte sur un monde, à la façon d'une "fenêtre" qui découpe la perspective fuyante d'un paysage offert » (Ricœur $1984: 189-190)$. Pour suggérer cette ouverture, Ricœur utilise ici une métaphore visuelle et picturale. De la même façon qu'une fenêtre crée une perspective sur le paysage, qui s'offre de l'autre côté, l'œuvre donne elle-aussi une perspective, une vue sur un monde qui la dépasse. À la conception structuraliste du texte comme structure close, autonome, Ricœur oppose l'idée que tout texte est ouvert, car tout texte est un discours. Or, un discours suppose que quelqu'un, donc un sujet, parle à quelqu'un d'autre pour lui dire quelque chose sur quelque chose. Ricœur développe ainsi une conception qui prend en compte à la fois l'auteur qui écrit l'œuvre, le lecteur réel (et non pas seulement le lecteur impliqué, c'est-à-dire la figure du lecteur tel qu'il apparaît dans le texte), le sens du texte (" dire quelque chose ») et sa référence, donc ce qui est visé par le sens («sur quelque chose»). La référence est recréée grâce à la lecture qui marque l'intersection entre le monde du texte et le monde du lecteur. Ainsi, à la configuration du texte en tant que structure correspond la refiguration du texte par le lecteur en termes d'expérience (Ricoeur 1985 : 309).

Ricœur distingue trois moments de l'acte de lecture auquel correspondent trois disciplines différentes: la stratégie de l'auteur dirigée vers le lecteur à qui il veut communiquer une vision du monde (objet de la rhétorique de la fiction développée par Booth), la réalisation de cette stratégie dans le texte (objet de la poétique) et la réponse du lecteur individuel (la phénoménologie de la lecture d'Iser) ou du lecteur comme collectivité (l'esthétique de la réception de Jauss). Ces trois modèles sont intégrés dans son concept de "refiguration ", qu'il interprète dans une perspective herméneutique, mobilisant notamment les notions de fusion d'horizons et d'application de Gadamer. Il s'intéresse d'abord à la stratégie de l'auteur qui crée une œuvre à travers laquelle il veut communiquer au lecteur une vision des choses. Il tient compte de l'auteur parce que le lecteur qui lit le texte perçoit toujours un projet de l'auteur. Ricœur ne parle pas de l'auteur réel mais de l'auteur impliqué, (notion qu'il emprunte à W. Booth), qui « disparait en se faisant narrateur immanent à l'œuvre - voix narrative » (Ricoeur 1985 : 310). La figure de l'auteur projette une figure du lecteur qui est un lecteur impliqué. Ricœur se demande s'il y a une symétrie entre les deux et sa réponse est non. Selon lui, la contrepartie de l'auteur impliqué n'est pas le lecteur impliqué mais le lecteur réel qui est « une concrétisation du lecteur impliqué, visé par la stratégie de persuasion du narrateur " (Ricœur 1985 : 310). Il définit la communication littéraire comme une communication asymétrique car «tandis que l'auteur réel s'efface dans l'auteur impliqué, le lecteur impliqué prend corps dans le lecteur réel » (Ricoeur 1985 : 310-311). 
19 Il est important de ne pas se méprendre sur la critique par Ricœur du paradigme structuraliste, lequel enfermerait le texte sur lui-même et ne tiendrait pas compte de sa référence. Il souligne en effet que si l'herméneutique est « l'art de discerner le discours

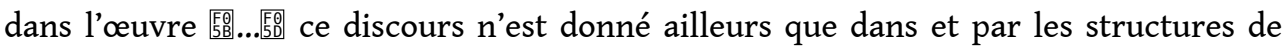
l'œuvre» (Ricoeur 1986: 124). Explication et compréhension, épistémologie et ontologie ne s'excluent pas mais se situent «sur un unique arc herméneutique » car « expliquer, c'est dégager la structure, c'est-à-dire les relations internes de dépendance qui constituent la statique du texte; interpréter, c'est prendre le chemin de pensée ouvert par le texte, se mettre en route vers l'orient du texte » (Ricoeur $1986: 175)$. Le passage par l'explication qui met le sens à distance, permet selon lui de passer d'une interprétation subjective, vue comme un "acte sur le texte", à une interprétation objective, vue comme un « acte du texte» (Ricoeur 1986 : 174-177). L'herméneutique de Ricœur apparaît ainsi comme un développement créateur des études narratives auxquelles il a conféré une nouvelle complexité.

Après avoir exposé sa théorie dans Temps et récit II, Ricœur la confronte à la pratique littéraire. Il analyse trois romans modernes, qui sont selon lui autant de «fables sur le temps ", à savoir Mrs. Dalloway de Virginia Woolf, La Montagne magique de Thomas Mann et À la recherche du temps perdu de Marcel Proust. Si Ricœur choisit ces trois romans, c'est parce que, selon lui, le temps y est non seulement un thème, mais opère comme un time-shift, comme quelque chose qui change la structure même du roman. Ces romans proposent des «variations imaginatives» sur le temps, «des variétés de l'expérience temporelle que seule la fiction peut explorer et qui sont offertes à la lecture en vue de refigurer la temporalité ordinaire » (Ricœur 1984 :191-192).

21 Pour préciser son approche des récits fictionnels, je me limiterai ici au commentaire d'un seul des trois romans, Mrs. Dalloway. Ricœur part de la configuration de l'œuvre, donc de l'analyse narratologique, et s'intéresse ensuite à la vision du monde et à «l'expérience temporelle que cette configuration projette hors d'elle-même » (1984: 192). Il souligne que c'est la configuration narrative qui « sert de support à l'expérience que ses personnages font du temps, et que la voix narrative veut communiquer au lecteur» (1984: 192). Le roman décrit une journée splendide de juin 1923, du matin jusqu'au soir, durant lequel aura lieu une réception donnée par Clarissa Dalloway, une femme de la haute société londonienne, ce qui marquera la fin du récit. Clarissa n'est pas le seul personnage central car «la mise en intrigue consiste à former une ellipse dont le second foyer est le jeune Septimus Warren Smith, un ancien combattant de la Grande Guerre, que sa folie conduit au suicide quelques heures avant que Clarissa ne donne sa réception » (1984:193).

Ricœur s'intéresse ensuite à la technique narrative de Virginia Woolf et à la façon dont est composé le roman. Le premier principe de composition est celui d'un « entassement progressif » (1984: 194) et « consiste à jalonner l'avancée de la journée par des menus événements" (1984:193) qui "tirent le récit vers sa clôture attendue» (1984: 194). Mais le récit est aussi «tiré en arrière, retardé en quelque sorte, par d'amples excursions dans le passé, qui constituent autant d'événements de pensée " (1984: 194-195). L'art de la fiction de Virginia Woolf consiste ainsi à tisser «ensemble le monde de l'action et celui de l'introspection » (1984:195-196), le présent et le souvenir du passé. Un deuxième procédé de la technique narrative de Mrs. Dalloway sur lequel Ricœur attire l'attention est le multiperspectivisme : le narrateur passe « d'un flux de conscience à l'autre, en faisant se rencontrer les personnages dans les mêmes lieux (les 
rues de Londres, le parc public), en les faisant assister aux mêmes incidents (le passage de la voiture du prince de Galles, le vol de l'aéroplane, etc.) » (1984: 197).

Ricœur passe ensuite de la phase explicative à la phase interprétative. Il montre ainsi " que ces procédés, caractéristiques de la configuration temporelle, servent à susciter le partage, entre le narrateur et le lecteur, d'une expérience temporelle ou plutôt d'une gamme d'expériences temporelles, donc à refigurer dans la lecture le temps lui-même ». (1984 : 199). Il étudie le rapport des différents personnages au temps chronologique marqué par les coups de Big Ben et des autres cloches qui sonnent l'heure. Selon Ricœur, «ce sont les variations de ce rapport, selon les personnages et les occasions, qui constituent l'expérience temporelle fictive que le récit construit avec un soin extrême » (1984: 199). Il met en évidence «la variété des rapports entre l'expérience temporelle concrète des divers personnages et le temps monumental» (1984: 204), allant de l'accord avec le temps monumental lié aux figures de l'autorité (incarnées par le Dr. Bradshaw) jusqu'à la terreur de l'histoire (incarnée par Septimus). Ricœur part du pôle de la discordance la plus radicale, à savoir «l'expérience de la mortelle discordance entre le temps intime et le temps monumental dont Septimus est le héros et la victime" (1984: 204). Les expériences temporelles des divers personnages s'organisent par rapport à la "faille insurmontable, creusée entre le temps monumental du monde et le temps mortel de l'âme» (1984: 207). L'expérience temporelle de Clarissa par rapport à celle de Septimus, de Peter et des figures de l'autorité «constitue l'enjeu du jeu avec le temps, opéré par les techniques narratives caractéristiques de Mrs. Dalloway " (1984: 208). Clarissa est à la fois du côté du temps monumental et un double de Septimus. Cependant, son temps n'est pas le temps de la mort, comme celui de Septimus, mais celui de l'affirmation de la vie. Ricœur souligne en dernière analyse que l'expérience du temps que propose Mrs. Dalloway est composée d'expériences différentes du temps qui sont reliées comme dans un réseau :

Cette expérience du temps n'est ni celle de Clarissa, ni celle de Septimus, ni celle de Peter, ni celle d'aucun des personnages: elle est suggérée au lecteur par le retentissement [...] d'une expérience solitaire dans une autre expérience solitaire. C'est ce réseau, pris dans son entièreté, qui est l'expérience du temps dans Mrs. Dalloway.

(Ricœur 1984 : 211-212)

Après avoir mis en évidence, à travers cet exemple, la façon dont Ricœur articule explication et interprétation, narratologie et herméneutique, je m'intéresserai dans ce qui suit à un autre dialogue, celui de la narratologie contemporaine avec Ricœur (donc avec l'herméneutique), mais aussi avec les sciences cognitives.

\section{De l'herméneutique à la narratologie cognitive : des mondes possibles à l'« expérientialité »}

Selon Raphaël Baroni, l'un des principaux mérites de Ricœur est non seulement d'avoir contribué à engendrer le "tournant narratif ", qui a eu lieu durant les années 1990, mais aussi d'avoir joué un rôle essentiel dans la transformation des études narratives en permettant de désenclaver la réflexion sur le récit du formalisme littéraire (Baroni $2010: 361)$.

Ricœur peut être vu comme un précurseur de la narratologie " postclassique » comme le montrent les travaux de Monika Fludernik, Raphaël Baroni ou Marco Caracciolo qui se réfèrent tous directement à lui ou partent de lui pour développer les possibilités de 
la narratologie. Il faut néanmoins ajouter que la plupart des narratologues se situent dans des cadres d'analyse qui diffèrent de la perspective herméneutique, à l'instar de la logique sémantique des mondes possibles, de la philosophie analytique, des sciences cognitives ou de la philosophie de l'esprit.

Herman, qui a introduit le concept de narratologie "postclassique ", souligne qu'elle " contient la narratologie classique comme un de ses "moments", mais [qu'] elle se caractérise par une profusion de nouvelles méthodologies et hypothèses de recherches » et qu' " en outre, dans sa phase postclassique, la recherche sur le récit ne se contente pas d'exposer les limites des modèles structuralistes anciens; elle en exploite aussi les possibilités " (Herman 1999 : 2-3). On peut observer en tout cas un déplacement de l'intérêt de la narratologie "postclassique » de l'analyse des structures textuelles vers l'étude de la façon dont les narrations façonnent ou remodèlent la réalité. Tout comme Ricœur avait mis en relation la préfiguration, la configuration et la refiguration, l'enjeu de cette narratologie est d'articuler les modèles cognitifs développés par la psychologie cognitive, la linguistique cognitive, les neurosciences ou la philosophie de l'esprit, avec l'analyse des structures narratives et avec l'expérientialité. Parmi cette pluralité d'approches, je mentionnerai ici celles qui semblent prolonger l'herméneutique de Ricœur, à savoir la narratologie cognitive et la narratologie transmédiale (Fludernik, Herman, Nünning, Ryan, Jahn).

La narratologie cognitive vise à élargir le champ de la narratologie en essayant de dialoguer avec les sciences cognitives (psychologie cognitive et neurosciences) (voir Herman 2013). Comme l'a souligné Jean-Marie Schaeffer l'étude cognitive des récits « déplace la question de l'analyse descriptive des récits publics vers celle des processus mentaux de production et de compréhension des récits " (Schaeffer 2020:32). La narratologie transmédiale quant à elle a élargi le champ du récit en s'intéressant non seulement aux récits littéraires, mais aussi à ceux véhiculés par d'autres médias, comme par exemple les récits visuels, audiovisuels ou interactifs (cinéma, jeux vidéo, etc.).

29 À l'instar de Ricœur dans Temps et récit, la narratologie "postclassique » a développé des concepts auxquels la narratologie structuraliste ne s'était pas intéressée ou qu'elle avait ignorés, comme le monde du texte, les univers fictionnels, l'expérience du lecteur. Elle a essayé de mettre en relation l'analyse des structures textuelles avec une prise en compte de la lecture et de l'expérience du lecteur. On observe ainsi un glissement du texte vers le monde du texte dans la réflexion de Marie-Laure Ryan (1991) sur les mondes possibles, ou chez David Herman (2013), ainsi qu'une ouverture du texte vers le lecteur (à travers la notion d'expérientialité) chez Monika Fludernik (2018) et Marco Caracciolo (2014b).

Dans « Les storyworlds au cœur des relations entre le récit et l'esprit », Herman (2018) montre que de nombreux travaux basés sur la narratologie structuraliste cherchent en même temps à "compléter» ou à "enrichir le répertoire de concepts hérités du structuralisme en prenant en compte la masse de recherches consacrées à l'intelligence humaine, restées inconnues ou inaccessibles aux narratologues classiques, afin d'éclairer les capacités et les dispositions mentales spécifiques qui fondent - ou au contraire sont fondées par - les expériences narratives » (2018: 95). Herman se propose ainsi d'explorer «les interfaces entre les récits et l'esprit» (2018: 96). Selon lui, la narratologie structuraliste a écarté le problème de la référentialité et de la modélisation des mondes par le récit parce qu'elle a pris comme modèle la linguistique 
de Saussure dont l'opposition signifiant/signifié exclut le référent. Herman soutient également que "le défi majeur pour les analystes du récit consiste à intégrer l'apport du récit comme ressource primordiale pour la modélisation et la création de mondes » (2018: 97). Il introduit la notion de storyworld, inspirée de la notion de narrative worldmaking développée par Goodman, et souligne que la construction des storyworlds est « une caractéristique propre des expériences narratives, une fonction primordiale des récits » $(2018: 96)$.

31 Herman analyse le récit à la fois en tant qu'objet d'interprétation, ce qu'il appelle « faire monde du récit » (2018:104), et comme moyen de donner un sens à l'expérience, ce qu'il définit comme « faire récit du monde » (2018: 108). Il souligne que « l'opération qui consiste à faire monde du récit implique l'utilisation d'indices textuels pour déterminer la configuration spatio-temporelle des événements narrés et pour construire l'ontologie du domaine narré - c'est-à-dire le modèle mental des entités qui existent dans le monde évoqué par le récit, accompagnées de leurs propriétés et de leurs relations " (2018: 104). L'analyse de Herman est narratologique parce que les storyworlds restent pour lui «des modèles plus ou moins détaillés permettant aux interprètes de formuler des inférences à propos des situations, des agents et des événements » (2018: 104).

Marie-Laure Ryan a développé quant à elle la question des mondes fictionnels comme mondes possibles (Ryan 1991) à la suite, entre autres, des travaux de Thomas Pavel et de Ruth Ronen. Elle s'est en particulier intéressée à la question de l'immersion dans Narrative as Virtual Reality: Immersion and Interactivity in Literature and Electronic Media, dans lequel elle développe une phénoménologie de la lecture distinguant l'immersion de l'interactivité, et la lecture passive de la lecture vue comme quelque chose d'actif, cette double orientation (la lecture comme figuration et comme configuration) apparaissant déjà chez Ricœur.

La notion d'« expérientialité » figure dans le Handbook of Narratology parmi les notions centrales de la narratologie contemporaine. Elle a été introduite par Monika Fludernik dans Towards a Natural Narratology (Fludernik 1996), où elle prend comme prototype de tout récit la narration «naturelle », ce par quoi elle entend les narrations orales, nonfictionnelles de la vie de tous les jours: les récits conversationnels. Fludernik rapproche sa démarche, qui consiste à partir des récits conversationnels (et non de la fiction littéraire) de celle d'Alfred Schütz, qui a utilisé la notion d'expérience quotidienne comme prototype des relations humaines dans le cadre de sa sociologie (Fludernik 1996 : 17).

Fludernik définit le concept de narrativité non plus en référence à l'intrigue ou à l'histoire, mais à l'expérientialité, qu'elle définit comme «l'évocation quasi-mimétique d'expériences de la vie réelle » (Fludernik 1996 : 12). Dans un article où elle revient sur son livre, elle souligne que l'expérientialité «fait référence à la dynamique entre la racontabilité et l'intérêt, constatée par Labov » (Fludernik 2018: 72) et qu'elle est «la capacité humaine à retravailler l'expérience en termes de signification émotive et évaluative » (Fludernik 2018: 75). Elle souligne ainsi que «l'ingrédient crucial [...] ne réside pas dans les séries d'actions narratives en elles-mêmes, mais dans leur surcharge expérientielle (émotionnelle et évaluative) » (2018: 72). Le modèle de Fludernik ancre donc la narrativité des narrations fictionnelles dans la représentation des expériences des personnages (1996: 30). Fludernik veut montrer, comme l'ont fait Käte Hamburger ou Dorrit Cohn, que le récit est la seule forme de discours capable de représenter la 
conscience d'une autre personne de l'intérieur (Fludernik 1996 : 27). Cependant, à la différence de Hamburger et Cohn, qui pensent que seul le récit fictionnel en est capable, pour Fludernik, c'est aussi l'apanage des récits "naturels", qui sont généralement factuels. Selon Fludernik, il peut y avoir des narrations sans intrigue, mais il n'y a pas des récits sans quelqu'un qui fait une expérience (experiencer) (Fludernik 1996: 13). Sa conception de la narrativité fondée sur l'expérientialité s'applique selon elle à la narration naturelle, tout autant qu'au drame ou au film, mais elle exclut l'histoire. Cette redéfinition de la narrativité entraîne ainsi une modification des frontières entre le narratif et le non-narratif. La poésie lyrique, qui n'est pas traditionnellement vue comme un récit, est considérée ici comme narrative, alors que l'historiographie, traditionnellement perçue comme une mise en récit de l'histoire, en est exclue, de même que les comptes rendus factuels, sous prétexte que ces discours ne se fondent par sur une représentation de l'expérientialité (Caracciolo 2014 : 150-151). Lorsque Fludernik évoque la question de l'historiographie, elle oppose sa conception à celle de Ricœur qui définit le récit à partir de la mise en intrigue :

Le facteur constitutif majeur du récit historique reste pour Ricoeur la mise en intrigue de l'évidence historique dans une histoire. Il montre ensuite que cette histoire est fictionnalisée d'une manière flagrante et dans une perspective clairement littéraire. Ricoeur ne fait curieusement pas de distinction entre l'agentivité d'un protagoniste fictionnel et celle d'un quasi-agent dans le discours historique. Une telle distinction serait fondée non pas sur les problèmes d'ordre cognitif concernant l'intentionnalité ou la réalisation de buts mais sur le caractère nécessairement expérientiel des événements affectant les agents nécessairement humains des récits de fiction, absent dans le cas des agents historiques dans le discours historique. (Fludernik $1996: 18$ )

Il me semble que la critique de Fludernik repose sur un malentendu. Ricœur trace une frontière nette entre fait et fiction, puisqu'il souligne que « seule l'historiographie peut revendiquer une référence qui s'inscrit dans l'empirie, dans la mesure où l'intentionnalité historique vise des événements qui ont effectivement eu lieu » (Ricœur 1983: 154). Il souligne ainsi qu' «à la différence du roman, les constructions de l'historien visent à être des reconstructions du passé ", qu' « à travers le document et au moyen de la preuve documentaire, l'historien est soumis à ce qui, un jour, fut » et qu'il a une dette à l'égard des morts (Ricoeur 1985 : 253). Même si la mise en forme littéraire est un trait commun aux deux types de récits (fictionnel et historique), Ricœur souligne que le but de l'historien est de représenter les choses telles qu'elles se sont passées, d'où sa critique de Hayden White. Il ne réduit donc pas l'histoire à une simple story, parce que l'histoire a aussi une dimension explicative. Par conséquent, la différence entre fiction et récit factuel qui intéresse Ricœur relève du mode de référentialité, et elle engage donc la question de la vérité.

Selon Fludernik, à l'instar des gens qui interprètent des expériences de la vie réelle en termes de schémas disponibles, les lecteurs construisent des significations et imposent des cadres à leur interprétation des textes (1996: 12). Elle montre ainsi que "l'expérientialité reflète un schéma cognitif d'incarnation (embodiedness) qui est lié à l'expérience humaine et à des préoccupations humaines " (1996: 30). Elle distingue quatre niveaux de cadres cognitifs. Le niveau expérientiel et cognitif de base (qui correspond selon elle à ce que Ricœur appelle mimèsis I) est celui des paramètres naturels de l'expérience de la vie réelle, à savoir la compréhension de l'intentionalité, des buts, des émotions, des causes, des effets. C'est à ce niveau que se situent les schèmes de la théorie des cadres de Schank et Abelson (frame theory). 
37 Au deuxième niveau, elle distingue cinq cadres ou paramètres cognitifs qui sont liés à la médiation narrative : "agir» « raconter», "faire l'expérience de », " percevoir ", " réfléchir ». Ils sont « liés à des perspectives fondamentales sur l'expérience humaine et sa médiation narrative » $(2018: 73)$ et définissent «des modes de transmission narrative à travers des types de perspective sur l'expérientialité engendrés par des schémas » (2018: 73). Le cadre de « l'action » est mobilisé lorsque le noyau expérientiel se présente "comme une série d'événements et de réactions vis-à-vis d'eux » (2018: 73), dans le cadre du « raconter » c'est « notre familiarité avec l'acte de raconter qui passe au premier plan » $(2018: 73)$. Le cadre de la «perception » « repose sur un mode cognitif qui conceptualise un spectateur présent sur la scène, qui observe les événements narratifs » (2018: 73). Le cadre «faire l'expérience de » se réfère au noyau expérientiel de la narration, qui est «centré sur l'immersion du protagoniste dans l'expérience » $(2018: 73)$. Enfin le cadre « réfléchir » « est lié à l'évaluation mentale de l'expérience » $(2018: 73)$.

Les paramètres cognitifs du troisième niveau se réfèrent à l'expérience pragmatique d'écouter ou de lire des histoires et principalement aux cadres littéraires/génériques de la narration. Le niveau 2 et 3 correspondent, selon Fludernik, non pas au niveau de la mimèsis II, mais, du moins en partie, à la mimèsis III. Au quatrième niveau se situe ce qu'elle appelle la narrativisation, qui est un processus dynamique engendré par l'expérience de la lecture. C'est en partant du concept de naturalisation de Culler que Fludernik introduit le concept de narrativisation pour « décrire une stratégie de lecture qui naturalise les textes en ayant recours à des schèmes narratifs » (Fludernik 1996 : 34). Le concept de naturalisation de Culler, se réfère aux stratégies interprétatives des lecteurs, lorsqu'ils sont confrontés à des incohérences : elles ont pour but de rendre familier, d'expliquer ce qui est étrange, insolite, déviant, en le rapportant à un autre cadre.

Fludernik utilise donc la notion d'expérientialité à la fois pour parler de l'expérience du narrateur, tel que figuré à l'intérieur du texte, et pour parler de l'expérience du lecteur, car «la fiction offre aux lecteurs des expériences qu'ils ne peuvent pas faire euxmêmes » (Fludernik 2018: 82). Selon Caracciolo, la définition de Fludernik oscille « entre l'orientation textualiste de la narratologie structuraliste et l'orientation vers le lecteur des approches postclassiques, notamment des approches cognitives » (Caracciolo 2014a: 152). Elle couvre ainsi «le continuum entre la représentation textuelle d'expériences fictionnelles et la création d'expériences induites par le récit chez les lecteurs » (2014a : 152). Certains narratologues comme Margolin et Palmer ont assimilé la notion d'« expérientialité » à la représentation textuelle de la conscience fictionnelle des personnages, qui est l'un des objets traditionnels de la narratologie (Hamburger 1986; Cohn 1981; Caracciolo 2014a: 151). D'autres, comme Caracciolo, prolongent la réflexion de Fludernik en étudiant l'expérientialité comme interaction entre le texte et le lecteur, se rapprochant du même coup de la perspective de Ricœur (cf. Caracciolo 2014a : 154).

40 Caracciolo part du même présupposé que Fludernik, à savoir l'hypothèse que la cognition est incarnée (embodied) (Caracciolo 2014b : 48), mais il considère l'embodiment comme une condition biologique, cognitive et existentiale qui sous-tend l'arrière-plan de l'expérience du lecteur. Dans la perspective de Fludernik, l'embodiedness « évoque tous les paramètres d'un schème d'existence de la vie réelle qui doit toujours être situé dans un cadre spatio-temporel spécifique » (Fludernik 1996:30). C'est parce qu'il voit 
l'expérience comme une expérience du monde incarnée (embodied) et évaluative que Caracciolo définit son approche comme "enactiviste» (Caracciolo 2014b: 97). Il s'inspire en particulier des travaux entrepris dans le domaine des sciences cognitives (Varela, Lakoff et Johnson), dans la philosophie de l'esprit (Gallagher et Zahavi) et dans la psychologie cognitive (Gerrig 2018).

Un deuxième trait commun à la démarche de Caracciolo et celle de Fludernik réside dans le fait qu'ils sont, l'un comme l'autre, redevables à la théorie de la triple mimèsis développée par Ricœur dans Temps et récit. Selon Caracciolo, la triple mimèsis de Ricœur correspond dans son modèle au répertoire ou arrière-plan de l'expérience des récepteurs (experiential background), au texte (avec les stratégies stylistiques et narratives adoptées par l'auteur) et à l'effet en retour du texte sur le répertoire luimême (Caracciolo 2014b: 48). Cependant, Caracciolo, à la différence de Fludernik, estime que l'expérientialité, vue comme relation mimétique entre récit et expérience humaine, n'est pas une relation à sens unique, dans laquelle les récits s'appuient uniquement sur la familiarité des récepteurs avec le monde réel, mais une relation dynamique dans laquelle le monde réel et les expériences induites par les récits interagissent (Caracciolo 2014b:153). L'engagement avec un récit exploite non seulement le répertoire des expériences passées des récepteurs (experiential background), mais il peut aussi entraîner des changements dans ce répertoire (2014b : 153). Comme pour Ricœur, l'expérience du lecteur apparaît ainsi comme une expérience transformatrice. Ce double mouvement entre storytelling et répertoire (background) est, selon Caracciolo, constitutif de l'expérientialité (Caracciolo 2014b: 154).

Selon Caracciolo l'expérientialité ne peut pas être comprise en termes représentationnels, mais devrait plutôt être conçue comme un réseau, qui résulte d'une double tension : celle entre la structure textuelle et l'arrière-plan de l'expérience du lecteur (experiential background) et celle entre l'attribution de l'expérience à un personnage et sa simulation imaginative ou son incarnation (enactement) par le récepteur (2014b : 49-50). L'expérientialité apparaît ainsi comme «la capacité d'une histoire d'exploiter et d'avoir un effet rétroactif sur l'arrière-plan (background) de différents récepteurs» et elle inclut par conséquent "la somme de toutes les expériences possibles induites par des récits » (2014b:50).

Si la plupart des études entreprises dans le cadre de la narratologie s'intéressent surtout à l'intersection entre mimèsis II et mimèsis III, d'autres travaux essaient d'élargir le cadre d'analyse de Ricœur, en partant de la mimèsis I. C'est le cas par exemple de Jean-Marie Schaeffer (2020) qui, dans Les Troubles du récit, s'intéresse à la mémoire épisodique, au rêve, à la planification des actions, ou encore aux activités imaginatives non fictionnelles qui modèlent l'identité individuelle et qui ont toutes, selon lui, une structure narrative, ou plutôt "proto-narrative ». Il définit la "proto-narrativité " comme "une forme spécifique d'organisation ou de traitement des représentations mentales" et la distingue à la fois de la "narration" - ce par quoi il entend «les productions narratives dans le cadre de la communication quotidienne interindividuelle » - et du récit - c'est-à-dire des productions narratives qui relèvent de l'art de raconter, et donc de l'art littéraire (Schaeffer 2020 : 46-47). Comme Ricœur, il établit un lien étroit entre la narrativité et l'identité personnelle, mais en l'ancrant à un niveau « sub-personnel » (Schaeffer 2020 :122, 132). 


\section{Conclusion}

Ce dialogue croisé entre l'herméneutique de Ricœur et la narratologie montre que la visée des deux disciplines n'est pas la même. L'intérêt de Ricœur pour le récit est de nature philosophique, car il voit en ce dernier une médiation qui permet de penser notre être dans le temps, notre identité (personnelle et collective) et notre relation à autrui. Le récit est capable selon lui de préfigurer, de configurer et de refigurer le temps, il fait la médiation entre le temps subjectif et le temps objectif. Le récit de fiction, grâce à ses variations imaginatives, permet d'explorer des mondes possibles dont on ne peut pas faire l'expérience autrement; il constitue un laboratoire de modèles existentiels divers. Il est aussi une médiation qui permet d'arriver à une meilleure compréhension de soi-même et des autres. L'objet principal de la narratologie, en revanche, ce sont d'abord les formes, les structures ou les bases cognitives du récit.

Pourtant le dialogue s'est avéré fructueux. Ricœur a ainsi tiré profit des travaux entrepris dans le champ de la narratologie structurale et a montré que la forme narrative est indissociable du contenu de l'œuvre. Il a souligné l'importance du passage par une phase explicative pour accéder au sens, ainsi que la nécessité d'articuler l'explication, c'est-à-dire l'analyse des structures, et l'interprétation, afin de dépsychologiser la compréhension. Sur un plan plus général, Ricœur montre que la littérature n'est pas un pur jeu formel, mais qu'elle a une référence, qu'elle nous dit quelque chose sur nous et sur le monde, qu'elle véhicule des valeurs.

La narratologie "postclassique » rejoint quant à elle sur bien des points la critique formulée par Ricœur à l'égard de la narratologie structurale. Elle s'intéresse aux mondes fictionnels et prend en compte le lecteur réel. Fludernik souligne par exemple que « le processus de lecture est fondamental pour la constitution de la narrativité » et que la narrativité n'est pas selon elle « une qualité inhérente au texte, mais un attribut imposé au texte par le lecteur qui l'interprète comme narratif, c'est-à-dire qui le narrativise» (Fludernik 2018: 70). Mais chez Fludernik, il y a encore une oscillation entre l'expérience du narrateur ou des personnages et l'expérience du lecteur. Caracciolo en revanche s'intéresse directement à l'expérience du lecteur. Sa conception entre ainsi fortement en résonance avec l'herméneutique de Ricœur.

En dépit de ces convergences, il est important de souligner que les problématiques de Ricœur et celles des narratologues ne coïncident pas totalement. Le concept de mimèsis III de Ricœur pose surtout le problème de la référence de la fiction et de l'histoire, alors que la notion d'expérientialité, inspirée par la linguistique cognitive, se réfère au fait que la narration est toujours médiatisée par une conscience, sans poser le problème de la vérité de la fiction. Chez Fludernik la notion d'expérientialité renvoie au fait que le récit est la représentation des expériences des personnages et permet ainsi aux lecteurs de faire des expériences qu'ils ne pourraient pas faire dans la vraie vie. Sur ce point, Fludernik semble rejoindre la conception de Ricœur. Cependant, l'expérience du lecteur telle que la pense Ricœur doit être comprise au sens gadamérien d'Erfahrung, c'est-à-dire comme une expérience capable de changer de façon fondamentale notre façon de nous comprendre et de comprendre le monde, donc comme une expérience transformatrice, et non comme quelque chose qui correspond à notre attente ou confirme ce que nous savons déjà (Gadamer 1996 : 376). Certes, Caracciolo essaie luiaussi de mettre en évidence cet aspect, mais sa notion d'expérience se réfère surtout à 
l'expérience simulée du lecteur. Il s'écarte en effet de la théorie des trois mimèsis de Ricœur, puisque les trois aspects qu'il identifie, à savoir l'arrière-plan (background) du lecteur, le texte, et l'effet en retour du texte sur cet arrière-plan, se réfèrent tous au processus de la lecture, donc uniquement à ce que Ricœur appelle mimèsis III, alors que ce dernier met en relation auteur, texte et lecteur. Quant au système de Fludernik, il ne prend pas en compte la mimèsis II, puisque le récit n'y est plus défini par la mise en intrigue mais par l'expérientialité.

\section{BIBLIOGRAPHIE}

Barthes, Roland (1966), « Introduction à l'analyse structurale des récits », Euvres complètes, tome II, Paris, Seuil.

Baroni, Raphaël (2010), «Ce que l'intrigue ajoute au temps. Une relecture critique de Temps et récit de Paul Ricœur », Poétique, n¹63, p. 361-382.

Caracciolo, Marco (2012), « Notes for A(nother) Theory of Experientiality », Journal of Literary Theory, $\mathrm{n}^{\circ}$ 6, p. 141-158.

Caracciolo, Marco (2014a), « Experientiality », in Handbook of Narratology, P. Hühn, J. C. Meister, J. Pier, W. Schmid (dir.), Berlin \& Boston, De Gruyter, vol. 1, p. 149-158.

Caracciolo, Marco (2014b), The Experientiality of Narrative. An Enactivist Approach, Berlin \& Boston, De Gruyter.

Cohn, Dorrit (1981), La Transparence intérieure, Paris, Seuil.

Fludernik, Monika (1996), Towards a Natural Narratology, London, Routledge.

Fludernik, Monika, (2018), « De la narratologie naturelle : une synthèse rétrospective », in Introduction à la narratologie postclassique, S. Patron (dir.), Villeneuve-d'Ascq, Presses Universitaires du Septentrion, p. 69-94.

Gadamer, Hans-Georg (1996 [1960]), Vérité et méthode, édition intégrale revue et complétée par Pierre Fruchon, Paris, Seuil.

Gallagher, Shaun, Zahavi, Daniel (2008), The Phenomenological Mind: An Introduction to Philosophy of Mind and Cognitive Science, Abingdon, Routledge.

Gerrig, Richard (1993), Experiencing Narrative Worlds: On the Psychological Activities of Reading, New Haven et Londres, Yale University Press.

Ginzburg, Carlo (2019 [1976]), Le Fromage et les Vers. L'univers d'un meunier du XVI siècle, traduit de l'italien par Monique Aymard, Paris, Flammarion.

Hamburger, Käte (1986), Logique des genres littéraires, Paris, Seuil.

Herman, David (1997), « Scripts, Sequences and Stories: Elements of a Postclassical Narratology », PMLA, n 112 (5), p. 1046-1059.

Herman, David (1999), «Introduction », in Narratologies: New Perspectives on Narrative Analysis, D. Herman (dir.), Columbus, The Ohio State University Press. 
Herman, David (2013), Storytelling and the Sciences of Mind, Cambridge, MIT Press.

Herman, David (2018), « Les storyworlds au cœur des relations entre le récit et l'esprit » in Introduction à la narratologie postclassique, S. Patron (dir.), Villeneuve-d'Ascq, Presses Universitaires du Septentrion, p. 95-119.

Lakoff, George \& Mark Johnson (1999), Philosophy in the Flesh: the Embodied Mind and Its Challenge to Western Thought, New York, Basic Books.

Levi, Giovanni, (1989), Le Pouvoir au village. Histoire d'un exorciste dans le Piémont du XVII siècle, traduit de l'italien par Monique Aymard, Paris, Gallimard.

Patron, Sylvie (dir.), Introduction à la narratologie postclassique. Les nouvelles directions de la recherche sur le récit, Villeneuve-d'Ascq, Presses Universitaires du Septentrion.

Pavel, Thomas (1988 [1986]), Univers de la fiction, Paris, Seuil.

Ricœur, Paul (1965), De l'interprétation. Essai sur Freud, Paris, Seuil.

Ricœur Paul (1969), Le Conflit des interprétations. Essais d'herméneutique, Paris, Seuil.

Ricœur, Paul (1983), Temps et récit I, L'intrigue et le temps historique, Paris, Seuil.

Ricœur, Paul (1984), Temps et récit II, La configuration du temps dans le récit de fiction, Paris, Seuil.

Ricœur, Paul (1985), Temps et récit III, Le temps raconté, Paris, Seuil.

Ricœur, Paul (1986), Du texte à l'action. Essais d'herméneutique, II, Paris, Seuil.

Ricœur, Paul (1988), « L’identité narrative », Esprit, n 7-8, p. 295-304.

Ricœur, Paul (1990), Soi-même comme un autre, Paris, Seuil.

Rimmon-Kenan, Shlommith (1989), « How the Model Neglects the Medium: Linguistics, Language and the Crisis of Narratology ", The Journal of Narrative Technique, $\mathrm{n}^{\circ} 19$ (1), p. 157-166.

Ryan, Marie-Laure (1991), Possible Worlds, Artificial Intelligence and Narrative Theory, Bloomington, Indiana University Press.

Ryan, Marie-Laure (2001), Narrative as Virtual Reality. Immersion and Interactivity in Literature and Electronic Media, Baltimore, John Hopkins University Press.

Ryan, Marie-Laure \& Jan-Noël Thon (2014), Storyworlds across Media. Towards a Media-Conscious Narratology, Lincoln, University of Nebraska Press.

Schaeffer, Jean-Marie (2010), « Le traitement cognitif de la narration », in Narratologies contemporaines, J. Pier \& F. Berthelot (dir.), Paris, Éditions des Archives contemporaines, p. 215-231.

Schaeffer, Jean-Marie (2020), Les Troubles du récit. Pour une nouvelle approche des processus narratifs, Paris, Éditions Thierry Marchaisse.

Schütz, Alfred (1960 [1932]), Der sinnhafte Aufbau der sozialen Welt. Eine Einleitung in die verstehende Soziologie, Berlin, Suhrkamp.

Strawson, Galen (2004), « Against Narrativity », Ratio, n 17, p. 428-452.

Varela, Francisco J., Evan Thompson, \& Eleanor Rosch (1991), The Embodied Mind: Cognitive Science and Human Experience, Cambridge, Massachusets, MIT Press.

Vultur, Ioana (2017), Comprendre. L'herméneutique et les sciences humaines, Paris, Gallimard.

Young, Kay \& Jeffrey L. Saver (2001), « The Neurology of Narrative », SubStance, n 94-95, p. 72-84. 


\section{RÉSUMÉS}

L'article porte sur le dialogue croisé entre Ricœur et la narratologie. D'une part, dans Temps et récit II, Ricœur est entré en débat avec la narratologie structuraliste, pour opposer à la fermeture du texte l'ouverture du discours, mais aussi pour l'intégrer à son propre modèle. Il a développé son modèle herméneutique du récit à partir de la narratologie (Genette), en introduisant la notion centrale de «monde du texte » à laquelle correspond, selon lui, «l'expérience fictive du lecteur ». D'autre part, le modèle de Ricœur a eu à son tour un impact sur la narratologie poststructuraliste, et en particulier sur la narratologie cognitive, qui a développé des possibilités qui se trouvaient in nuce dans Temps et récit et a tenté elle aussi de mettre en relation l'analyse des structures textuelles avec une prise en compte de la lecture et de l'expérience du lecteur. La notion centrale d'« expérientialité » introduite par Monika Fludernik reprend la discussion de l'articulation entre intrigue et expérience dans le cadre de la narratologie.

The article focuses on the cross-dialogue between Ricœur and narratology. On the one hand, Ricoeur entered into a debate with structuralist narratology, not only in order to oppose the openess of discourse to the closure of the text, but also in order to integrate narratology into his own model. He developed his hermeneutical model taking narratology as a departure point (Genette), by introducing the central notion of "world of the text " to which corresponds, according to him, a « fictive experience of the reader ». On the other hand, Ricœur's model had an impact on poststructuralist narratology, particularly on cognitive narratology, which developed possibilities contained in nuce in Time and narrative and also tried to connect the analysis of textual structures with the experience of the reader. The central notion of " experientiality" introduced by Monika Fludernik, replaces the debate of the articulation between plot and experience in the context of narratology.

\section{INDEX}

Mots-clés : Ricœur, herméneutique, narratologie, récit, mise en intrigue, monde du texte, expérience du lecteur, expérientialité, sciences cognitives

\section{AUTEUR}

\section{IOANA VULTUR}

Ioana Vultur est spécialiste de littérature française et comparée. Elle a soutenu une thèse de doctorat à l'Université Paris IV-Sorbonne, intitulée « Temps et remémoration dans A la recherche du temps perdu de M. Proust et La Mort de Virgile de H. Broch » sous la direction d'Antoine Compagnon. Elle a eu un contrat postdoctoral à l'EHESS (Paris), où elle a enseigné pendant plusieurs années dans le cadre du Master « Arts et langage », puis à la Freie Universität de Berlin (Dahlem Research School). Elle s'est ensuite occupée de la coordination scientifique du projet de recherche «Création, cognition, société » (Université Paris Sciences et Lettres). Elle a publié des articles et deux livres : Proust et Broch : les frontières du temps, les frontières de la mémoire, (Paris, L'Harmattan, 2003) et Comprendre. L'herméneutique et les sciences humaines (Paris, Gallimard, 2017). En 2015, elle a dirigé un numéro de la revue Critique « Où va l'herméneutique ?». Elle vient de codiriger un numéro de Communications sur « Les arts et les âges de la vie » (à paraître en octobre 2021). 